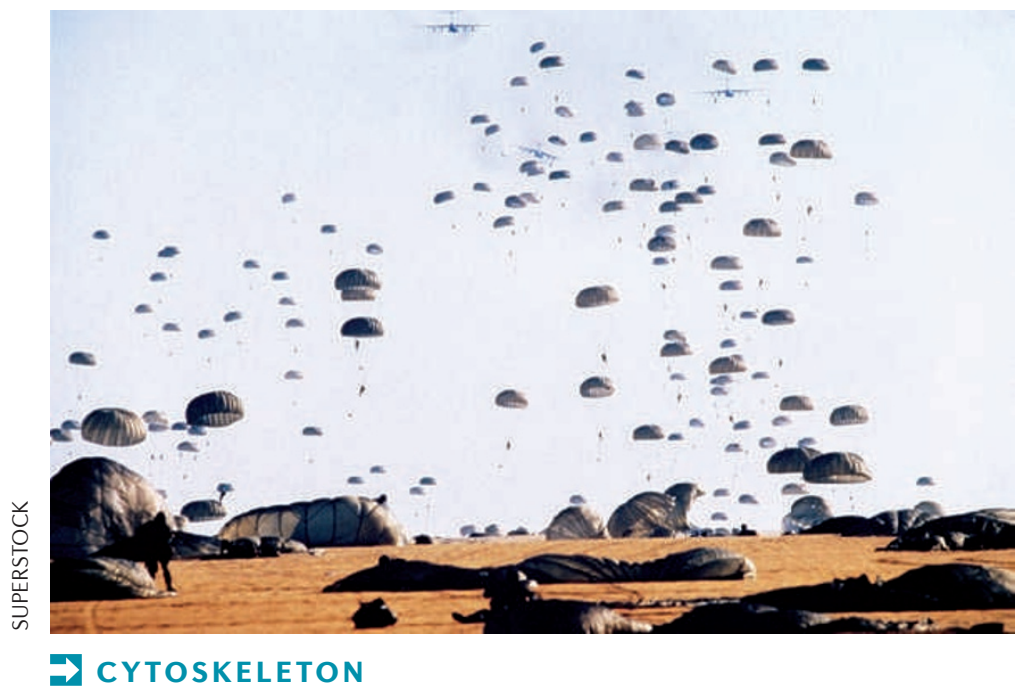

\section{Invading forces}

Although it has been appreciated for some time among cell biologists that proteins that regulate the geometry of the actin cytoskeleton can affect cell motility, less is known about the roles these proteins might have in cancer. John Condeelis, Frank Gertler and colleagues show that the Enabled (Ena)/vasodilatorstimulated phosphoprotein (Vasp) family member MENA, which binds to and regulates actin, can promote invasion and metastasis of breast cancer cells.

MENA has several splice variants, one of which is more highly expressed in invasive mammary tumour cells in rats and mice $\left(\mathrm{MENA}^{\mathrm{INV}}\right)$. Rat mammary tumour cells (MTLn3) expressing enhanced green fluorescent protein (EGFP)tagged MENA or MENA ${ }^{\mathrm{INV}}$ were injected orthotopically into rat mammary glands, and intravital imaging of the tumours that formed revealed that MENA and MENA ${ }^{\text {INV }}$ localized to the leading edge of motile tumour cells. Their expression increased the percentage of motile cells in vivo, but significantly more motile cells were seen with expression of MENA ${ }^{\text {INV }}$ compared with MENA. Xenografts of these cells in the mammary glands of severe combined immunodeficient mice demonstrated that the observed differences in motility induced by MENA and MENA ${ }^{\mathrm{INV}}$ were mirrored by increases in metastasis to the lungs.

How does MENA ${ }^{\mathrm{INV}}$ increase motility and invasion? In vitro collagen gel invasion assays of MTLn3 cells showed that the F-actin and profilin binding sites of MENA ${ }^{\mathrm{INV}}$ are crucial, indicating that increased invasion is probably a result of effects on actin dynamics. Consequently, MENA and MENA ${ }^{\text {INV }}$ seem to enhance the stability and therefore longevity of invadopodia in these three-dimensional assays, thus allowing increased matrix degradation by local activation of proteases. Actin filament nucleation by the ARP2-ARP3 complex and severing of actin filaments to create free barbed ends by cofilin are both required to create lamellipodia in two-dimensional assays. MENA ${ }^{\text {INV }}$ recruitment to the leading edge was dependent on free barbed ends, and cells expressing MENA ${ }^{\mathrm{INV}}$ had more cofilin-generated free barbed ends, leading to increased actin polymerization and lamellipodia extension. Recruitment of MENA ${ }^{\mathrm{INV}}$ preceded that of ARP2-ARP3, suggesting that ARP2-ARP3 is not required for MENA $^{\text {INV }}$ targeting.

MTLn3 cells usually require macrophages, which secrete epidermal growth factor (EGF), to invade collagen gels. Expression of MENA ${ }^{\mathrm{INV}}$, and to some extent MENA, allowed the cells to invade in the absence of macrophages, in response to only a small concentration of EGF that was present in the culture medium. This sensitization to EGF also occurred in vivo, indicating that MENA $^{\text {INV }}$ directs the response to EGF. No changes were observed in the phosphorylation of EGF receptor (EGFR), of proteins downstream of EGFR such as Erk and Akt, or of MENA $^{\mathrm{INV}}$ itself.

The authors propose a model in which MENA ${ }^{\mathrm{INV}}$ facilitates elongation of cofilin-generated barbed ends that subsequently serve as substrates for ARP2-ARP3 mediated nucleation and branching.

Sarah Seton-Rogers

ORIGINAL RESEARCH PAPER Philippar, U. et al.

A Mena invasion isoform potentiates EGF-

induced carcinoma cell invasion and metastasis. Dev. Cell 15, 813-828 (2008)

FURTHER READING Wang, W., Eddy, R. \&

Condeelis, J. The cofilin pathway in breast cancer invasion and metastasis. Nature Rev. Cancer 7 , 429-440 (2007) 